\title{
Preservation of Uncultivated Microbial Cells for Single Cell Genomics and Cultivation in Future
}

\author{
Om Prakash* and Kamlesh Jangid \\ Microbial Culture Collection, National Centre for Cell Science, Sai Trinity, Pashan, Pune- 411021, Maharashtra, India
}

\section{Short Commentary}

Microbes have conventionally been studied in pure culture obtained on culture media in the laboratory. With very limited media options at the disposal of a microbiologist as compared to the vast diversity of microbes that are known to exist, it is not surprising that only about $1-10 \%$ of them have been cultivated so far [1]. However, since microbes are the key components of every process on the planet, the cultivation of these as-yet-uncultivated microbes and the conservation of microbial diversity is the current research priority of the world for sustainable environment and Earth. Metagenomic studies prove beyond doubt that the vast majority of this yet-uncultivated microbial diversity inhabiting our planet has immense medical, agricultural, environmental and industrial potential. In order to utilize this hidden potential, cultivation and appropriate preservation of the source material is the only viable option. Driven by this fact, microbiologists have started seeking these yet-uncultivated microbes using high-throughput approaches of microbial cultivation giving rise to what is now known as culturomics [2]. While culturing was rampant during the infancy of microbiology, the recent rebirth of culturomics has successfully brought some previously uncultured microbes into the laboratory through innovative strategies. Many of these involved the use of different carbon sources and/or electron acceptors in the medium, extended incubation in a wide range of $\mathrm{pH}$ and temperature conditions, and growth in laboratory conditions that mimic the natural environment, such as in diffusion chambers or attached to carriers [3]. However, it is still a big challenge to cultivate all sorts of organisms and preserve them in culture collections for future use and study [4]. The availability of a live culture will supplement the information made available from metagenomics studies on the physiology, functionality and industrial applications of the organism. Although culturomics is time consuming and labour intensive, it has raised the hope that with careful design of culture media and cultivation conditions it is possible to bring the asyet-uncultivated microbes in culture for their study and exploitation.

Information gleaned from laboratory-adapted microbes may not represent the true nature of its wild state. Almost all established techniques of microbial cultivation, isolation and purification of microbes are based on cell populations, which are clones of a single cell produced after multiple divisions or generations. In addition to the accumulation of preservation-induced mutations, cells are known to acquire somatic variations or cellular heterogeneity during cell division and multiplication. With more prominance at the transcriptome level, cellular heterogeneities may go undetected in most metagenomic studies. Further, due to lack of established protocols for handling of single cells, and isolation and error-free amplification of DNA from a single cell, most data on the physiology, functionality, genomes and transcriptomes of microbes are available from cell populations, which neglects these variations acquired during growth and may therefore misrepresent the actual processes going on at single cell levels [5]. The need to study microbes at single cell is now emerging into an organized approach through single cell genomics. Advances in single cell separation methods like micro-pipetting, fluorescence activated cell sorting (FACS), microfluidics and microdisections methods [5] and use of whole genome amplification method using the multiple displacement amplification polymerase chain reaction [6], development of high throughput next generation sequencing and advance methods in bioinformatics have all made single cell genomics more precise and easy to carry out which was not possible a few years ago [5,7].

It was generally considered that unlike plants and animals, microbes are omnipresent and do not face extinction and endanger. However, recent developments in microbial ecology and metagenomics have shown that microbial cells have also undergone changes due to the effect of climate variation. Therefore, the preservation of microbial diversity, especially from highly susceptible ecosystems and extreme environments such as hot springs and thermal vents, is necessary to prevent the loss of valuable microbial diversity due to climatic change and environmental perturbation and serves as a prerequisite for future cultivation, single cell genomics and metagenomic purposes. Ex-situ preservation of cultivated microbes has almost been optimized except for a few groups of organisms like non-spore forming fungi and some fastidious bacteria $[1,4]$. Currently, all culture collections are dealing with cultivated pure cultures and preservation research related to uncultivated-microbial single cells, mixed cultures and microbiomes is still very limited. It is thus extremely important to preserve uncultivated microbes, which are facing the problem of extinction or climatic variation, in their natural state until strategies are developed to grow them in pure culture in the laboratory.

The question now arises as to which preservation strategy should be used, optimized and promoted to preserve such microbial diversity? Extraction of total genomic DNA and its preservation for future investigation, such as comparative metagenomics or to study the effect of environmental factors on microbial community structure is one such strategy. On the other hand, preservation of the environmental sample of the valuable habitat with an intact microbiome for future cultivation and study of the genome and/or proteome of single cells or the entire population is another good strategy. In addition, gentle separation of microbial cells or biomass from the environmental matrix without damage followed by the preservation of intact microbiome or single cells is another good option. Considering the need and importance of preservation of mixed microbial cultures (microbiome), intact environmental samples and single cells for single cell genomics, efforts have already been initiated in this direction. Kerckhof et al.

*Corresponding author: Om Prakash, Microbial Culture Collection, Nationa Centre for Cell Science, Sai Trinity, Pashan, Pune- 411021, Maharashtra, India, Tel: +91-20-25329036; Fax: +91-20-25329001; E-mail: prakas1974@gmail.com

Received July 25, 2015; Accepted July 28, 2015; Published July 31, 2015

Citation: Prakash O, Jangid K (2015) Preservation of Uncultivated Microbia Cells for Single Cell Genomics and Cultivation in Future. Single Cell Biol S1: 003. doi:10.4172/2168-9431.S1-003

Copyright: (c) 2015 Prakash O, et al. This is an open-access article distributed under the terms of the Creative Commons Attribution License, which permits unrestricted use, distribution, and reproduction in any medium, provided the original author and source are credited. 
(2014) studied the cryopreservation of methanotrophic co-culture; [8] oxygen limited autotrophic nitrification/denitrification biofilm and faecal sample (human microbiome) and showed good preservation of functionality and structure[8]. In addition, Morono et al. (2015) tried to optimize the preservation of intact environment sample using the cell alive system (CAS) freezing technique and found that the recovery of cells from the CAS-preserved sample was more efficient than preserved using conventional freezing methods [9]. In a similar study, Clingenpeel et al. (2014) found that cryopreservation of samples of three bacterial strains allowed better recovery of single cell genomes as compared to ethanol and paraformaldehyde preserved cell samples [10]. Despite the fact that the strategies are still developing, the potential benefits of preserving environmental samples with their communities are immeasurable.

In conclusion, the rebirth of culturomics has raised hopes of cultivating the yet-uncultivated microbes. While metagenomics has revolutionized the field of microbial ecology by allowing studying communities in details, the specific information that single-cell genomes are able to provide, is not possible with metagenomics alone. However, each field may supplement each-other and together they will be very useful, especially for investigating the microbial dark matter $[11,12]$ and bringing them to the laboratory culture. Single cell genomics, proteomics and transcriptomics of yet-uncultivated microbes is possible due to advancements in single cell separation, sequencing technology and bioinformatics. But the study of single cell genome and cultivation of known but yet-uncultivated diversity is both expensive and time consuming. In addition, due to the huge microbial diversity and also variation in habitats, single cell genomics of fresh culture and application of cultivation strategies for all the samples will be tough. This requires continuous development and optimization of preservation strategies for intact environmental samples, mixed cultures (microcosms) and single cells for future study and use.

\section{Conflict of Interest}

There is no conflict amongst the authors of this manuscript.

\section{Acknowledgement}

This work was supported by the Department of Biotechnology (DBT; Grant no. BT/PR/0054/NDB/52/94/2007), Government of India, under the project "Establishment of microbial culture collection".

\section{References}

1. Prakash O, Nimonkar Y, Shouche YS (2013) Practice and prospects of microbial preservation. FEMS Microbiol Lett 339: 1-9.

2. Lagier, JC, Hugon P, Khelaifia S, Fournier PE, La Scola B, et al. (2015) The rebirth of culture in microbiology through the example of culturomics to study human gut microbiota. Clin Microbiol Rev 28: 237-264.

3. Alain K, Querellou J (2009) Cultivating the uncultured: limits, advances and future challenges. Extremophiles 13: 583-594.

4. Prakash O, Shouche YS, Jangid K (2013) Microbial cultivation and the role of microbial resource centers in the omics era. Appl Microbiol Biotechnol 53 247-252.

5. Macaulay IC, Voet T (2014) Single cell genomics: advances and future perspectives. PLoS Genetics 10: e1004126.

6. Linnarsson S (2013) Single-cell biology meeting marks rebirth of an old science. Genome Biol 14: 305.

7. Lasken RS (2012) Genomic sequencing of uncultured microorganisms from single cells. Nature Rev Microbiol 10: 631-640.

8. Kerckhof FM, Courtens ENP, Geirnaert A, Hoefman S, Ho A, et al. (2014) Optimized cryopreservation of mixed microbial communities for conserved functionality and diversity. PLoS ONE 9: e99517.

9. Morono Y, Terada T, Yamamoto Y, Xiao N, Hirose T, et al. (2015) Intact preservation of environmental samples by freezing under an alternating magnetic field. Environ Microbiol Rep 7: 243-251.

10. Clingenpeel S, Schwientek P, Hugenholtz P, Woyke T (2014) Effects of sample treatments on genome recovery via single-cell genomics. The ISME J 8: 2546 2549 .

11. Hedlund B, Doszworth JA, Staley JT (2015) The changing landscape of microbial biodiversity exploration and its implications for systematics. Syst Appl Microbiol 38: 231-236.

12. Jahn M, Seifert J, Hübschmann T, von Bergen M, Harms H, et al. (2013) Comparison of preservation methods for bacterial cells in cytomics and proteomics. J Integrated OMICS 3: 25-33. 